This item was submitted to Loughborough's Research Repository by the author.

Items in Figshare are protected by copyright, with all rights reserved, unless otherwise indicated.

\title{
Augmented vagal heart rate modulation in active hypoestrogenic pre- menopausal women with functional hypothalamic amenorrhoea
}

\section{PLEASE CITE THE PUBLISHED VERSION}

http://dx.doi.org/10.1042/cs20150209

\section{PUBLISHER}

(c) Authors; published by Portland Press Limited

\section{VERSION}

AM (Accepted Manuscript)

\section{PUBLISHER STATEMENT}

This work is made available according to the conditions of the Creative Commons Attribution-NonCommercialNoDerivatives 4.0 International (CC BY-NC-ND 4.0) licence. Full details of this licence are available at: https://creativecommons.org/licenses/by-nc-nd/4.0/

\section{LICENCE}

CC BY-NC-ND 4.0

\section{REPOSITORY RECORD}

O'Donnell, Emma, Jack M. Goodman, Beverley L. Morris, John S. Floras, and Paula J. Harvey. 2019. "Augmented Vagal Heart Rate Modulation in Active Hypoestrogenic Pre-menopausal Women with Functional Hypothalamic Amenorrhoea". figshare. https://hdl.handle.net/2134/20606. 


\title{
Augmented Vagal Heart Rate Modulation in Active Hypoestrogenic Premenopausal Women with Functional Hypothalamic Amenorrhea
}

\author{
Emma O'Donnell ${ }^{1}$, Jack M Goodman ${ }^{1,2}$, Beverly L Morris ${ }^{3}$, John S Floras ${ }^{2,3}$, \\ and Paula J Harvey ${ }^{4}$
}

${ }^{1}$ Cardiovascular Research Laboratory, Department of Exercise Sciences, University of Toronto, Toronto, Ontario, Canada, and Department of Medicine, Division of Cardiology at the ${ }^{2}$ Mount Sinai Hospital, ${ }^{3}$ Toronto General Hospital, and ${ }^{4}$ Women's College Hospital, University of Toronto, Ontario, Canada.

\author{
Corresponding Author \\ Dr Paula J Harvey, BMBS, PhD, FRACP \\ Division of Cardiology, Women's College Hospital, \\ Department of Medicine, University of Toronto, \\ 76 Grenville Street \\ Toronto, Ontario, \\ M5S 1B3 \\ Canada \\ Tel: 416-323-7722 \\ Fax: 416-323-6304 \\ Paula.harvey@wchospital.ca
}

Running Title: Heart Rate Modulation in Amenorrheic Women

Key words: autonomic nervous system; energy deficiency; estrogen; exercise; heart rate variability.

Word Count: 4102 (excluding tables, figures, figure legends and references) 


\begin{abstract}
Compared with eumenorrheic women, exercise-trained women with functional hypothalamic amenorrhea (ExFHA) exhibit low heart rates (HR) and absent reflex reninangiotensin-system activation and augmentation of their muscle sympathetic nerve response to orthostatic stress. To test the hypothesis that their autonomic HR modulation is altered concurrently, three age-matched (pooled mean, $24 \pm 1$ years; mean \pm SEM) groups of women were studied: active with either FHA (ExFHA; $n=11$ ) or eumenorrheic cycles (ExOv; $n=17)$, and sedentary with eumenorrheic cycles (SedOv; $\mathrm{n}=17)$. Blood pressure (BP), HR, and HR variability (HRV) in the frequency domain were determined during both supine rest and graded lower body negative pressure (LBNP; 10, -20, and -40mmHg). Very low (VLF), low (LF) and high (HF) frequency power spectra $\left(\mathrm{ms}^{2}\right)$ were determined and, due to skewness, $\log _{10}$ transformed. LF/HF ratio and Total power (VLF+LF+HF) were calculated. At baseline, HR and systolic BP were lower $(p<0.05)$, and HF and Total power higher $(p<0.05)$ in ExFHA than eumenorrheic women. In all groups, LBNP decreased $(p<0.05)$ systolic BP, HF and Total power, and increased $(p<0.05) \mathrm{HR}$, and LF/HF ratio. However, HF and Total power remained higher $(p<0.05)$, and HR, systolic BP and LF/HF ratio lower $(p<0.05)$ in ExFHA than eumenorrheic women, in whom measures did not differ $(p>0.05)$. At each stage, HR correlated inversely $(p<0.05)$ with HF. In conclusion, ExFHA women demonstrate augmented vagal yet unchanged sympathetic HR modulation, both at rest and during orthostatic stress. Although the role of estrogen deficiency per se is unclear, these findings contrast with studies reporting decreased HRV in hypoestrogenic postmenopausal women.
\end{abstract}




\section{INTRODUCTION}

Functional hypothalamic amenorrhea (FHA) is a reversible cause of premenopausal ovarian suppression. FHA is characterized by chronically low levels of circulating estradiol that resemble those observed in postmenopausal women and men [1,2]. The prevalence of FHA is markedly higher in active ( $1-44 \%)$ versus sedentary $(-2-5 \%)$ women [3], and has been causally related to energy deficiency due to high energy expenditure (i.e., exercise) and insufficient caloric intake [4]. During simulated orthostatic stress, exercise trained women with FHA (ExFHA) demonstrate lower heart rate $(\mathrm{HR})$ and systolic blood pressure, augmented reflex sympathetic outflow to muscle yet absent reflex increases in plasma renin and angiotensin II compared with their eumenorrheic counterpart [5]. These observations suggest that cardiac autonomic regulation may also be altered in ExFHA women.

$\mathrm{HR}$ variability (HRV) provides both insight into cardiac vagal and sympathetic heart rate modulation and prognostic information [6]. Increased HRV is associated with decreased risk of cardiovascular events [6]. In contrast, low HRV attributed to elevated sympathetic modulation of HR predicts increased risk of cardiac arrhythmias postmyocardial infarction [6] and a higher risk of fatal and non-fatal cardiovascular events even in apparently healthy individuals $[7,8]$. Estrogen deficiency in postmenopausal women is associated with diminished HRV as a consequence of increased sympathetic and/or decreased vagal tone $[9,10]$. Conversely, exercise trained hypoestrogenic postmenopausal women exhibit higher HRV and greater cardiac vagal tone compared to their sedentary counterpart $[9,10]$. The influence of estrogen deficiency on HRV in exercise-trained premenopausal women with FHA is unknown.

The purpose of this study was to investigate the consequences of hypoestrogenemia in physically active premenopausal women with FHA for HR regulation. Therefore, in estrogen deficient ExFHA women and estrogen-replete exercise-trained and sedentary eumenorrheic women, we compared HRV both at rest and during an orthostatic hypotensive challenge (lower body negative pressure, LBNP) to reflexively increase HR. We anticipated, from the literature $[11,12]$, that HR would be lower in ExFHA women compared with eumenorrheic women, both at rest and during LBNP, and hypothesized this would be due to greater parasympathetic modulation.

\section{METHODS}

Subjects: Volunteers were recruited by posters targeting both sedentary and physically active premenopausal women. Screening procedures included general questionnaires on exercise, eating behaviour, menstrual cycle, and medical health. Eligibility criteria for the study included: 1) age 18 to $35 \mathrm{yrs}$; 2) absence of chronic illness, including diabetes, hyperprolactinemia, poly-cystic ovarian syndrome, and thyroid disease; 3) stable menstrual status over the preceding 3 months (i.e., either absence of menses or menstrual cycles between 25-35 days); 4) not taking any mediations; 5) non-smoker; 6) not currently dieting and weight stable for the preceding 3 months; 7 ) no history or current clinical diagnosis of eating disorders; and 8) absence of hormonal therapy for at least 6 months. The study was carried out in accordance with the Declaration of Helsinki (2008) of the World Medical Association, and the study was approved by the local University and Hospital Research Ethics Boards. All volunteers provided written informed consent.

Experimental Design: Volunteers were recruited consecutively over three years to participate in a larger cross-sectional study examining the cardiovascular consequences of estrogen deficiency in premenopausal women. Fifty-seven women participated in the 
larger study, which comprised three smaller studies. Participants enrolled in 1-3 of these smaller studies. In the current paper, subject characteristic data, and heart rate and blood pressure recorded at rest and during LBNP for ExOv and ExFHA groups include, in part, data from participants that have previously been reported by our group [5,11]. The present study reports novel data on our primary outcome variable, HRV, which was assessed at rest and during LBNP in forty-one of the 57 women.

Exercise and Menstrual Status Definitions: Volunteers were grouped according to exercise status (exercising or sedentary) and menstrual/ovulatory status (eumenorrheic and ovulatory, or amenorrheic). To conform with previously published convention with respect to studies in such women, exercise status was defined as "sedentary" when purposeful exercise was $<2$ hours per week and "exercising" when purposeful exercise was conducted $>2$ hours per week [13]. Further, based on literature describing 'average' peak aerobic capacity between 38-42 in sedentary women [14] a cut' off of $\leq 40$ $\mathrm{ml} / \mathrm{kg} / \mathrm{min}$ defined "sedentary" status and $>40 \mathrm{ml} / \mathrm{kg} / \mathrm{min}$ reflected "exercising" status. Eumenorrhea was defined as 10-13 menstrual cycles per year. Ovulation, confirmed during the menstrual cycle prior to testing, was detected using a urinary ovulation hormone kit (Clearblue Easy ovulation test, Unipath Diagnostics, Waltham, MA). FHA in association with exercise training was defined as cessation of menses for 90 or more consecutive days [3].

Study Groups: Three study groups were established: recreationally exercise trained women with FHA (ExFHA; $n=12)$ or eumenorrheic ovulatory menstrual cycles (ExOv; $n=14$ ), and sedentary women with eumenorrheic ovulatory menstrual cycles (SedOv; $n=15$ ). To examine the effects of estrogen deficiency in physically active women on HRV, values in amenorrheic physically active women with FHA were compared to those observed in eumenorrheic ovulatory estrogen-replete physically active women. Sedentary eumenorrheic ovulatory estrogen-replete physically active women were recruited as a 'normal' healthy reference group.

Subject Preparation: All measures were obtained during the early follicular phase (low estrogen and progesterone phase; days 2-6) of the menstrual cycle in eumenorrheic subjects, and on a random day for amenorrheic women. Thus, we were able to compare the cardiovascular effects of cyclically low estrogen (eumenorrheic women) versus chronically low estrogen (FHA women) levels. All tests occurred in the morning between 0930-1030 in a quiet ambient temperature room $\left(22-24^{\circ} \mathrm{C}\right)$. Volunteers had fasted at least 2 hours and abstained from alcohol 12 hours, and caffeine and exercise for 24 hours, prior to testing.

Anthropometric Measures and Body Composition: Total body mass and height were determined using a physician's balance scale (Detecto, Webb City, MO). Body composition was determined using dual-energy x-ray absorptiometry (DXA; Prodigy, General Electric Lunar Corporation, Madison, WI).

Peak Aerobic Capacity: On a separate study day, peak aerobic capacity $\left(\mathrm{VO}_{2}\right.$ peak) was measured using a metabolic cart during a progressive treadmill test to exhaustion (Moxus Modular $\mathrm{VO}_{2}$ System, Applied Electrochemistry Inc., Pittsburgh, PA).

Blood Sampling: Using standard venipuncture techniques, blood samples were collected at baseline for determination of serum $17 \beta$-estradiol, progesterone, testosterone, and sex hormone binding globulin (SHBG). On a separate day, 8-hour 
fasted serum free triiodothyronine (T3) was also assessed to provide an estimate of energy status, with low T3 levels indicating low energy status (i.e., energy deficiency) [15]. Free androgen index ([total testosterone/SHBG] ${ }^{\star} 100$ ) was calculated to provide an estimate of androgenicity [16]. All assays were run by the Core Laboratory at the Toronto General Hospital.

Blood Pressure and Heart Rate: Measurements of brachial systolic (SBP), diastolic (DBP), mean arterial BP (MAP) and HR were recorded from the left upper-arm using an automated device (Dinamap Pro 100, Critikon, USA). Brachial BP and HR were assessed at one minute intervals at baseline (three consecutive stable measures) and every minute throughout each stage of LBNP. The recordings acquired during the last four minutes of each LBNP stage were averaged to acquire a mean value for each LBNP stage. Continuous recordings of HR and BP were also acquired using lead II of an electrocardiogram and a photoplethysmographic device on the index finger (Portapres Model-2, Finapres Medical Systems BV, USA), respectively.

Heart Rate Variability: Frequency domain analysis of HRV was performed as described previously [17]. In brief, 7-minute recordings of continuous HR intervals (R-R) were collected using lead II of an ECG during spontaneous breathing at baseline and during each stage of LBNP. The ECG signal was sampled at $1000 \mathrm{~Hz}$ and was stored using LabView (National Instruments, Austin, TX) for off-line analysis. Frequency domain analysis was performed using a nonparametric method of fast Fourier transformation (FFT). Each 7-minute data set comprised 2,048 data points which were divided into seven segments, each containing 512 points, with one-half overlapping of each segment. The linear trend in the data was subtracted from the data set in each segment, and a Blackman-Harris window was applied to minimize spectral leakage. The power spectrum was subsequently quantified into very low (VLF: $0.001-0.05 \mathrm{~Hz}$ ), low (LF: 0.05 to $0.15 \mathrm{~Hz}$ ) and high (HF: 0.15 to $0.5 \mathrm{~Hz}$ ) frequencies. Frequency domains were determined in absolute units $\left(\mathrm{ms}^{2}\right)$, and as per Task Force recommendations [18] \{, $1996 \# 106\}$, HF and LF power were both transformed $\left(\log _{10}\right)$ and normalized (\%; LFnu=LF power/(total power-VLF power) $\times 100$, and HFnu=HF power/(total power-VLF power) $x$ 100). HF power is considered an index of vagal HR modulation, and LF power is considered an index of primarily sympathetic modulation, with some contribution from the efferent vagus [18]. The LF/HF ratio has been proposed as a rough estimate of cardiac sympatho-vagal balance [18]. For all data sets, only stationary time series with $\leq 5 \%$ arrhythmia or artefact were used for analyses.

LBNP: With subjects in the supine position, the lower body was encased in a custom built chamber that was sealed at the level of the iliac crest [19]. The chamber was attached to a vacuum source to effect reductions in pressure. HR, BP, respiratory excursions and HRV were continuously recorded at baseline and during each 8-minute stage of LBNP $(-10,-20$, and $-40 \mathrm{mmHg})$. Each LBNP stage was followed by 5-minutes of recovery (i.e., no LBNP). LBNP was terminated if SBP declined to $<80 \mathrm{mmHg}$, pallor was observed, and/or subjective feelings of nausea, dizziness and/or light-headedness were reported.

Statistics: All data sets were tested for non-normality, homogeneity of variance, and outliers. Consistent with previous literature [18], raw HRV data were non-normally distributed. Thus, both normalized and log10 converted LF and HF raw data were calculated. At baseline, between-group differences were detected using one-way 
analysis of variance (ANOVA), and when a significant main (fixed) effect was observed, within-group and between-group analyses of responses to LBNP, including change $(\Delta)$ in measures compared to baseline, were determined using mixed model ANOVA, using GROUP as the between-group factor. When significant within-group and between-group differences were detected, Bonferroni methods were used to determine where the significant differences existed. When assumptions of sphericity were violated, the Greenhouse-Geisser correction was used. Using pooled data, Pearson's correlational analyses were used to determine significant linear independent associations between HRV indices and variables of interest. Data were analyzed using packaged software (SPSS version 20; SPSS Inc., Chicago, IL). All data are presented as the mean \pm SEM. A significance level of $p<0.05$ was used to detect the differences for statistical procedures.

\section{RESULTS}

Subject Characteristics: Groups did not differ ( $p>0.05)$ in age, height, weight, or body composition (Table 1). Sedentary women were significantly less aerobically conditioned $(p<0.001)$ compared with exercising women. Serum measures of $17 \beta$-estradiol $(\mathrm{pmol} / \mathrm{L})$ did not differ ( $p>0.05)$ between ovulatory groups. In contrast, $17 \beta$-estradiol was lower $(p<0.05)$ in ExFHA compared with ExOv only. Progesterone was similar $(p>0.05)$ between groups $(1.8 \pm 0.7 \mathrm{nmol} / \mathrm{L}$; pooled mean). Free T3 $(\mathrm{pmol} / \mathrm{L})$ was significantly lower $(p<0.05)$ in ExFHA compared with ExOv and SedOv women. Testosterone and FAI did not differ $(p>0.05)$ between groups. SHBG was lower $(p<0.05)$ in SedOv versus ExOv and ExFHA women.

Blood Pressure and Heart Rate: Baseline Values: Between groups, HR and SBP were lower $(p<0.05)$ in ExFHA women than in SedOv and ExOv women, in whom HR and SBP did not differ ( $p>0.05$; Table 2). Baseline DBP was similar $(p>0.05)$ between the groups.

LBNP Responses: Within groups, graded LBNP elicited a significant increase $(p<0.05)$ in HR, and a decrease $(p<0.05)$ in SBP and DBP. Between-groups, analyses showed that HR and SBP values during LBNP remained significantly lower $(p<0.05)$ in ExFHA compared with SedOv and ExOv women. DBP values during LBNP did not differ ( $p>0.05$, main effect) between the groups. No between-group differences for BP and HR values were detected during graded LBNP for SedOv and ExOv women ( $p>0.05)$. Delta values (data not shown) for all HR and BP responses did not differ $(p>0.05)$ between all groups.

Heart Rate Variability: Baseline Values: Complete HRV data were unavailable in one SedOv woman whose study was terminated when presyncopal symptoms were evoked by $-20 \mathrm{mmHg}$ LBNP. ExFHA women demonstrated higher $(p<0.05$; main effect) baseline HF $\left(\mathrm{ms}^{2}\right)$, Total HRV, and HFlog10 compared with SedOv and ExOv women (Table 3). LF/HF ratio trended lower $(p=0.095)$ in ExFHA women than ovulatory women. In contrast, groups did not differ $(p>0.05)$ with respect to baseline VLF $\left(\mathrm{ms}^{2}\right)$, LF $\left(\mathrm{ms}^{2}\right)$,

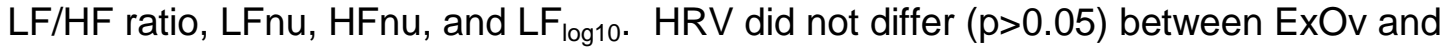
SedOv women.

LBNP Responses: Within-groups, LBNP elicited significant reductions in Total $\operatorname{HRV}(F(1.92,82.66)=7.175, \mathrm{p}=0.002$; main effect $), \mathrm{HFnu}(F(2.31,99.29)=46.54$, $\mathrm{p}<0.001)$, and $\mathrm{HF}_{\log 10}(F(1.63,70.18)=72.411, \mathrm{p}<0.001$; Figure 1). In contrast, LF/HF ratio $(F(1.42,61.07)=30.156, p<0.001)$, and $\operatorname{LFnu}(F(2.29,102.92)=44.239, \mathrm{p}<0.001)$ were increased by LBNP. VLF $\left(\mathrm{ms}^{2}\right), \operatorname{LF}\left(\mathrm{ms}^{2}\right)$, and $\mathrm{LF}_{\mathrm{log} 10}\left(\mathrm{~ms}^{2}\right)$ were unaltered $(p>0.05)$ by LBNP. No LBNP x Group interactions $(p>0.05)$ were detected. Between- 
groups, ExFHA women demonstrated significantly higher Total HRV ( $p=0.001$; main effect), HFnu ( $p<0.001$; main effect) and $\mathrm{HF}_{\log 10}(p<0.001$; main effect) at each LBNP stage compared with SedOv and ExOv women. Conversely, LF/HF ratio was significantly lower ( $p=0.023$; main effect) at $-20 \mathrm{mmHg}$ and $-40 \mathrm{mmHg}$ in ExFHA versus SedOv women only. HRV did not differ $(p>0.05)$ between SedOv and ExOv women at any LBNP stage. Delta HRV responses to LBNP did not differ $(p>0.05)$ between the groups.

Respiration Rate: Respiration rate did not differ ( $p>0.05$; main effect) between groups at baseline (14.2 \pm 0.4 breaths/minute; overall baseline mean $\pm S E M)$, nor was it altered by LBNP ( $p>0.05$, main effect).

Correlates of HR and HRV: Resting HR was positively associated with $17 \beta$-estradiol $(r=0.517, p=0.001)$ and T3 $(r=0.611, p<0.001)$. In contrast, baseline measures of $L_{\log 10}$ $(r=-0.028), \mathrm{HF}_{\log 10}(r=-0.299)$ and Total HRV $(r=-0.164)$ were not associated $(p>0.05)$ with serum measures of $17 \beta$-estradiol. No resting measure for HRV correlated with T3 (all $p>0.05$ ).

\section{DISCUSSION}

To our knowledge, this is the first study to evaluate autonomic heart rate modulation using frequency domain analysis of heart rate variability in estrogen deficient exercise trained premenopausal women with functional hypothalamic amenorrhea. We report that compared with age-matched eumenorrheic sedentary and exercise-trained estrogen replete women, exercise-trained estrogen deficient premenopausal women have lower heart rate both at rest and during orthostatic stress. LBNP reflexively increased heart rate in all groups in association with lowered HF, unaltered LF and increased LF/HF ratio. However, hypoestrogenic exercise trained premenopausal women demonstrated higher HF and Total power values at rest and during orthostatic stress compared with their exercise-trained and sedentary eumenorrheic peers. LF/HF ratio was also significantly lower in amenorrheic than eumenorrheic sedentary women during LBNP. Collectively, these observations are consistent with the concept that otherwise healthy hypoestrogenic exercise-trained premenopausal women have augmented vagal HR modulation, resulting in greater total HRV spectral power.

\section{Estrogen and Autonomic HR Modulation}

Several lines of evidence support the concept that estrogen modulates HR and cardiac autonomic tone. For example, premenopausal women demonstrate higher basal cardiac vagal tone compared with age-matched males $[20,21]$ and postmenopausal women [21]. Estrogen therapy lowers resting HR in normotensive postmenopausal women [22], and increases vagal and/or decreases sympathetic HR modulation at rest in most $[20,21]$ but not all [23] postmenopausal women. The effects of fluctuating estrogen levels across menstrual cycle on HRV are less clear, likely in part due to differences in the timing of data collection across the cycle. Notwithstanding, studies variably report greater [24,25] and unaltered [26] HRV during the follicular (increasing estrogen, low progesterone) versus the mid-luteal (high estrogen and progesterone) phase. In animals, $17 \beta$ estradiol administration increases parasympathetic and decreases sympathetic modulation of HR in both male and ovariectomized rodents [27]. The expression of significant populations of both estrogen receptor-alpha and -beta on cell bodies, axons and terminals of autonomic regulatory nuclei throughout the neuraxis is thought to explain in part the autonomic effects of estrogen [28]. 
In contrast with the literature in postmenopausal women, we report that despite hypoestrogenemia, LF HRV did not differ between the three study groups, yet vagal modulation of HR was enhanced both at rest and during LBNP in ExFHA women. Further, LF/HF ratio was lower during -20 and $-40 \mathrm{mmHg}$ LBNP in ExFHA versus sedentary eumenorrheic women, suggesting that their reflex sympathetic HR response to this stimulus may also be attenuated. An alternate interpretation is that the parasympathetic response was reflected in both the numerator and denominator of this ratio. It has been demonstrated that LF is not purely an index of cardiac sympathetic modulation, rather it is a marker of both sympathetic and vagal heart rate modulation, including modulation by the arterial baroreflex [29]. The supine position, as utilized in the current study, is also associated with greater vagal values compared with the seated or standing position [30]. Thus, it is possible that in the current study supine LF HRV, and indeed LF/HF, was primarily influenced by parasympathetic modulation. Because of this complexity, HF rather than LF was our principal focus of investigation in this experiment.

Collectively, low resting HR and augmented HRV in ExFHA women at rest and during LBNP suggests that these findings are not a direct consequence of estrogen deficiency. In light of the etiology of FHA in physically active women, alternative factors contributing to the elevated parasympathetic modulation of HR in ExFHA may include energy deficiency (i.e., caloric deficit), aerobic exercise training, and low brain and circulating angiotensin II concentrations, all of which are independently associated with augmented vagal modulation of $\mathrm{HR}[9,10,31,32]$. The possible contribution of these three factors to the observed enhanced HRV in ExFHA women are considered below.

\section{Energy Deficiency and HR Modulation}

Amenorrhea in weight-stable active women is causally linked to mild negative energy balance in association with increased energy expenditure that is frequently combined with subtle deficits in caloric intake [4]. In response to energy deficiency, circulating T3 levels are decreased in ExFHA women [33]. In accordance with this, we report low T3 in ExFHA women compared with eumenorrheic women. T3 is known to exert positive modulatory effects on HR by binding primarily to thyroid receptor- $\alpha$ expressed in the heart and the vasculature, eliciting genomic and non-genomic actions that influence gene transcription and peripheral hemodynamics [34]. As previously reported by our group [33], we observed a strong positive association between circulating levels of T3 and HR. In contrast, we did not observe a correlation between T3 and any index of HRV. This observation is perhaps not too surprising since the contemporary literature concerning thyroid hormones and heart rate variability has yet to achieve consensus. For example, the influence of the parasympathetic nervous system on HR is reported as being reduced in both hypo- and hyperthyroidism, with pharmacological restoration of euthyroid status eliciting increases in cardiac vagal tone $[35,36]$. Conversely, in both human and animal studies, low T3 elicited by caloric restriction alone has been correlated positively with HRV due to increased HF and/or decreased LF power [34].

\section{Exercise Training and HR Modulation}

Aerobic conditioning in humans is associated with resting bradycardia and increased HRV with greater resting parasympathetic and less sympathetic efferent heart rate modulation $[9,10]$. Alterations in the intrinsic sino-atrial node pacemaker [37] and neurotransmission at the level of the nucleus tractus solitarius [38] have been implicated in these changes. In the current study, we report similar cardio-respiratory fitness in our exercising groups yet lower HR and higher vagal modulation of HR both at rest and during LBNP in ExFHA versus ExOv women. In active young women high-volume and - 
intensity exercise training elicits greater vagal modulation of HR at rest and during postural stress compared with women undertaking low-intensity and -volume exercise training [39], and high-intensity exercise training leads to greater cardiorespiratory fitness than moderate- or low-intensity exercise training [40]. With the two active groups having similar fitness, it is therefore unlikely that exercise training intensity per se can account for the augmented vagal modulation of HR in ExFHA women. Interestingly, the present finding is consistent with reports of augmented cardiac vagal modulation of HR in aerobically trained ovariectomized versus intact rats [41,42], suggesting that hypoestrogenemia may modulate exercise-training induced effects on parasympathetic HR modulation. Examination of such postulates awaits delineation.

Angiotensin II and HR Modulation: Recently, our group reported that compared with estrogen replete ExOv women, ExFHA women demonstrate an absence of reflex activation of circulating renin and angiotensin II in response to orthostatic stress [5] \{O'Donnell, $2015 \# 1343$ \}. Substantial evidence links the renin-angiotensin-aldosterone system and the sympathetic nervous system $[32,43,44]$. Interactions can occur at central sites, such as in the rostral ventrolateral medulla and hypothalamus, and at the sympathetic prejunctional angiotensin II type 1 receptor, which facilitates norepinephrine release from the sympathetic nerve terminal $[32,43,44]$. Blockade of the angiotensin II type 1 receptor decreases sympathetic modulation of HR in experimental heart failure [44] and administration of angiotensin converting enzyme inhibitors increases vagal modulation of HR in patients after acute myocardial infarction [43]. Thus, angiotensin II exerts both sympatho-stimulatory and vagal-inhibitory effects on HR modulation [32]. Of note, angiotensin II infusion has been reported to elicit sex-specific cardiac autonomic responses, with healthy young men exhibiting a shift toward sympathovagal imbalance due to withdrawal of parasympathetic activity, and healthy young women demonstrating unaltered sympathovagal balance [45]. Whether resistance to the vagolytic effects of angiotensin II infusion is diminished in functionally hypoestrogenemic women, is unknown. However, our previous finding of significantly lower circulating angiotensin II concentrations at -20 and $-40 \mathrm{mmHg}$ in ExFHA (2.1 \pm 0.3 and $2.0 \pm 0.4 \mathrm{pmol} / \mathrm{L}$, respectively) versus ExOv women (5.3 \pm 1.4 and $8.6 \pm 1.9 \mathrm{pmol} / \mathrm{L}$, respectively) [5] suggests that low circulating angiotensin II in ExFHA women during orthostatic stress may contribute to their augmented cardiac parasympathetic tone during LBNP.

Limitations: Respiratory sinus arrhythmia, or the HF (vagal) component of HRV, is influenced by both the depth and frequency of respiration [46]. Despite having similar breathing frequencies ( 14 breaths per minute) among our study groups, it is possible that women with ExFHA may have different tidal volumes, which were not recorded. Due to the low prevalence ( 2-5\%) [3] and the psychogenic- and/or weight loss-related origins of FHA in sedentary women [47], our investigations did not extend to the effects of premenopausal estrogen deficiency on HRV in such women. Therefore, our study findings are limited to the effects of estrogen deficiency combined with exercise training on HRV.

Conclusion: Estrogen is assumed to elicit cardioprotective effects on the autonomic nervous system, including elevated HRV in association with increased vagal and decreased sympathetic modulation of HR [21]. In contrast to this concept, we report that physically active estrogen deficient premenopausal women with FHA demonstrate elevated HRV as a consequence of increased vagal HR modulation both at rest and during graded orthostatic stress. Further, HRV is elevated in ExFHA women above that observed in fitness-matched estrogen replete women. While the influence of estrogen 
deficiency per se on cardiac autonomic modulation in ExFHA women is unclear, these findings suggest complex interactions between competing factors may oppose the cardiac autonomic modulatory effects of estrogen deficiency. In light of the etiology of FHA in active women, such factors likely include low circulating levels of triiodothyronine, angiotensin II, and exercise training-estrogen interactions.

\section{Acknowledgements}

$\mathrm{PJH}$ was funded by a Pfizer Cardiovascular Independent Research Award (NRA 3840028). JSF is a Canada Research Chair in Integrative Cardiovascular Biology. We acknowledge and thank all the members of the Clinical Cardiovascular Physiology Laboratory at the Toronto General Hospital for their technical assistance and collaboration. We would also like to thank each of our participants for their time and efforts. 


\section{References}

1. Elmlinger, M.W., Kuhnel, W., and Ranke, M.B. (2002) Reference ranges for serum concentrations of lutropin (LH), follitropin ( $F S H)$, estradiol (E2), prolactin, progesterone, sex hormone-binding globulin (SHBG), dehydroepiandrosterone sulfate (DHEAS), cortisol and ferritin in neonates, children and young adults. Clin. Chem. Lab. Med. 40, 1151-1160

2. O'Donnell, E., Goodman, J.M., and Harvey, P.J. (2011) Cardiovascular consequences of ovarian disruption: a focus on functional hypothalamic amenorrhea in physically active women. J. Clin. Endocrinol. Metab. 96, 36383648

3. Otis, C.L., Drinkwater, B., Johnson, M., Loucks, A., and Wilmore, J. (1997) American College of Sports Medicine position stand. The female athlete triad. Med. Sci. Sports Exercise 29, i-ix

4. Williams, N.I., Helmreich, D.L., Parfitt, D.B., Caston-Balderrama, A., and Cameron J.L. (2001) Evidence for a causal role of low energy availability in the induction of menstrual cycle disturbances during strenuous exercise training. J. Clin. Endocrinol. Metab. 86, 5184-5193

5. O'Donnell, E., Goodman, J.M., Mak, S., Floras, J.S., Morris, B.L., and Harvey, P.J. (2015) Discordant orthostatic reflex renin-angiotensin and sympathoneural responses in premenopausal exercising-hypoestrogenic women. Hypertension 65, 1089-1095

6. La Rovere, M.T., Pinna, G.D., Hohnloser, S.H., Marcus, F.I., Mortara, A., Nohara, R., Bigger, J.T. Jr., Camm, A.J., and Schwartz, P.J. (2001) Baroreflex sensitivity and heart rate variability in the identification of patients at risk for life-threatening arrhythmias: implications for clinical trials. Circulation 103, 2072-2077

7. Galinier, M., Pathak, A., Fourcade, J., Androdias, C., Curnier, D., Varnous, S., Boveda, S., Massabuau, P., Fauvel, M., Senard, J.M., and Bounhoure, J.P. (2000) Depressed low frequency power of heart rate variability as an independent predictor of sudden death in chronic heart failure. Eur. Heart J. 21, 475-482

8. Hillebrand, S., Gast, K.B., de Mutsert, R., Swenne, C.A., Jukema, J.W., Middeldorp, S., Rosendaal, F.R., and Dekkers, O.M. (2013) Heart rate variability and first cardiovascular event in populations without known cardiovascular disease: meta-analysis and dose-response meta-regression. Europace 15:742749

9. Davy, K.P., DeSouza, C.A., Jones, P.P., Seals, D.R. (1998) Elevated heart rate variability in physically active young and older adult women. Clin. Sci. 94, 579584

10. Davy, K.P., Miniclier, N.L., Taylor, J.A., Stevenson, E.T., and Seals, D.R. (1996) Elevated heart rate variability in physically active postmenopausal women: a cardioprotective effect? Am. J. Physiol. 271, H455-460

11. O'Donnell, E., Goodman, J.M., Mak, S., and Harvey, P.J. (2014) Impaired vascular function in physically active premenopausal women with functional hypothalamic amenorrhea is associated with low shear stress and increased vascular tone. J. Clin. Endocrinol. Metab. 99, 1798-1806

12. O'Donnell, E., Harvey, P.J., Goodman, J.M., and De Souza, M.J. (2007) Longterm estrogen deficiency lowers regional blood flow, resting systolic blood pressure, and heart rate in exercising premenopausal women. Am. J. Physiol. Endocrinol. Metab. 292, E1401-1409

13. De Souza, M.J., Miller, B.E,. Loucks, A.B., Luciano, A.A., Pescatello, L.S., Campbell, C.G., and Lasley B.L. (1998) High frequency of luteal phase deficiency 
and anovulation in recreational women runners: blunted elevation in folliclestimulating hormone observed during luteal-follicular transition. J. Clin.

Endocronol. Metab. 83, 4220-4232

14. Willmore J.H, and Costll D.L. (2005) Physiology of Sport and Exercise. 3rd ed. Champaign, IL: Human Kinetics.

15. Loucks, A.B., and Heath, E.M. (1994) Induction of low-T3 syndrome in exercising women occurs at a threshold of energy availability. Am. J. Physiol. 266, R817823

16. Rickenlund, A., Carlstrom, K., Ekblom, B., Brismar, T.B., von Schoultz, B., and Hirschberg, A.L. (2003) Hyperandrogenicity is an alternative mechanism underlying oligomenorrhea or amenorrhea in female athletes and may improve physical performance. Fertil. Steril. 79, 947-955

17. Notarius, C.F., Butler, G.C., Ando, S., Pollard, M.J., Senn, B.L., and Floras, J.S. (1999) Dissociation between microneurographic and heart rate variability estimates of sympathetic tone in normal subjects and patients with heart failure. Clin. Sci. 96, 557-565

18. Task force of the European Society of Cardiology and the North American Society of Pacing Electrophysiology. (1996) Heart rate variability. Standards of measurement, physiological interpretation and clinical use. Circulation 93, 10431065

19. Harvey, P.J., Morris, B.L., Miller, J.A., and Floras, J.S. (2005) Estradiol induces discordant angiotensin and blood pressure responses to orthostasis in healthy postmenopausal women. Hypertension 45, 399-405

20. Huikuri, H.V., Pikkujamsa, S.M., Airaksinen, K.E., Ikaheimo, M.J., Rantala, A.O., Kauma, H., Lilja, M., and Kesaniemi, Y.A. (1996) Sex-related differences in autonomic modulation of heart rate in middle-aged subjects. Circulation 94, 122125

21. Liu, C.C., Kuo, T.B., and Yang, C.C. (2003) Effects of estrogen on gender-related autonomic differences in humans. Am. J. Physiol. Heart Circ. Physiol. 285, $\mathrm{H} 2188-2193$

22. Harvey, P.J., Wing, L.M., Savage, J., and Molloy, D. (1999) The effects of different types and doses of oestrogen replacement therapy on clinic and ambulatory blood pressure and the renin-angiotensin system in normotensive postmenopausal women. J. Hypertens. 17, 405-411

23. Vrtovec, B., Starc, V., and Meden-Vrtovec, H. (2001) The effect of estrogen replacement therapy on ventricular repolarization dynamics in healthy postmenopausal women. J. Electrocardiol. 34, 277-283

24. Bai, X., Li, J., Zhou, L., and Li, X. (2009) Influence of the menstrual cycle on nonlinear properties of heart rate variability in young women. Am. J. Physiol. Heart Circ. Physiol. 297, H765-774

25. Yildirir, A., Kabakci, G., Akgul, E., Tokgozoglu, L., and Oto, A. (2002) Effects of menstrual cycle on cardiac autonomic innervation as assessed by heart rate variability. Ann. Noninvasive Electrocardiol. 7, 60-63

26. Leicht, A.S., Hirning, D.A., and Allen, G.D. (2003) Heart rate variability and endogenous sex hormones during the menstrual cycle in young women. Exp. Physiol. 88, 441-446

27. Saleh, T.M., and Connell, B.J. (1999) Centrally mediated effect of 17betaestradiol on parasympathetic tone in male rats. Am. J. Physiol. 276, R474-481

28. Saleh, T.M., and Connell, B.J. (2007) Role of oestrogen in the central regulation of autonomic function. Clin. Exp. Pharmacol. Physiol. 34, 827-832 
29. Reyes del Paso, G.A., Langewitz, W., Mulder, L.J., van Roon, A., and Duschek, S. (2013) The utility of low frequency heart rate variability as an index of sympathetic cardiac tone: a review with emphasis on a reanalysis of previous studies. Psychophysiol. 50, 477-487

30. Cacioppo, J.T., Berntson, G.G., Binkley, P.F., Quigley, K.S., Uchino, B.N., and Fieldstone, A. (2007) Autonomic cardiac control. II. Noninvasive indices and basal response as revealed by autonomic blockades. Psychophysiol. 31, 586598

31. De Angelis, K., Wichi, R.B., Jesus, W.R., Moreira, E.D., Morris, M., Krieger, E.M. and Irigoyen, M.C. (2004) Exercise training changes autonomic cardiovascular balance in mice. J. Appl. Physiol. 96, 2174-2178

32. Reid, I.A. (1992) Interactions between ANG II, sympathetic nervous system, and baroreceptor reflexes in regulation of blood pressure. Am. J. Physiol. 262, E763778

33. O'Donnell, E., Harvey, P.J., and De Souza M.J. (2009) Relationships between vascular resistance and energy deficiency, nutritional status and oxidative stress in oestrogen deficient physically active women. Clin. Endocrinol. 70, 294-302

34. Kahaly, G.J., and Dillmann, W.H. (2005) Thyroid hormone action in the heart. Endocr. Rev. 26, 704-728

35. Cacciatori, V., Gemma, M.L., Bellavere, F., Castello, R., De Gregori, M.E., Zoppini, G., Thomaseth, K., Moghetti, P., and Muggeo, M. (2000) Power spectral analysis of heart rate in hypothyroidism. Euro. J. Endocrinol. 143, 327-333

36. Chen, J.L., Chiu, H.W., Tseng, Y.J., and Chu, W.C. (2006) Hyperthyroidism is characterized by both increased sympathetic and decreased vagal modulation of heart rate: evidence from spectral analysis of heart rate variability. Clin.

Endocrinol. 64, 611-616

37. Negrao, C.E., Moreira, E.D., Santos, M.C., Farah, V.M., Krieger, E.M. (1992) Vagal function impairment after exercise training. J. Appl. Physiol. 72, 1749-1753

38. Mueller, P.J., and Hasser, E.M. (2006) Putative role of the NTS in alterations in neural control of the circulation following exercise training in rats. Am. J. Physiol. Regul. Integr. Comp. Physiol. 290, R383-392

39. Gilder, M., and Ramsbottom, R. (2008) Change in heart rate variability following orthostasis relates to volume of exercise in healthy women. Auton. Neurosci. 143, 73-76

40. Wenger, H.A., and Bell, G.J. (1986) The interactions of intensity, frequency and duration of exercise training in altering cardiorespiratory fitness. Sports Med. 3, 346-356

41. Silveira, L.C., Tezini, G.C., Schujmann, D.S., Porto, J.M., Rossi, B.R., and Souza, H.C. (2011) Comparison of the effects of aerobic and resistance training on cardiac autonomic adaptations in ovariectomized rats. Auton. Neurosci. 162, 35-41

42. Tezini, G.C., Silveira, L.C., Villa-Cle, P.G., Jr., Jacinto, C.P., Di Sacco, T.H., and Souza, H.C. (2009) The effect of aerobic physical training on cardiac autonomic control of rats submitted to ovariectomy. Menopause. 16, 110-116

43. Kontopoulos, A.G., Athyros, V.G., Papageorgiou, A.A., Skeberis, V.M., Basayiannis, E.C., and Boudoulas, H. (1997) Effect of angiotensin-converting enzyme inhibitors on the power spectrum of heart rate variability in postmyocardial infarction patients. Coron. Artery Dis. 8, 517-524

44. Murakami, H., Liu, J.L., and Zucker I.H. (1996) Blockade of AT1 receptors enhances baroreflex control of heart rate in conscious rabbits with heart failure. Am. J. Physiol. 271, R303-309 
45. Mann, MC., Exner, D.V., Hemmelgarn, B.R., Turin, T.C., Sola, D.Y., and Ahmed, S.B. (2012) Impact of gender on the cardiac autonomic response to angiotensin II in healthy humans. J. Appl. Physiol. 112, 1001-1007

46. Kobayashi, H. (1998) Normalization of respiratory sinus arrhythmia by factoring in tidal volume. Appl. Human Sci. 17, 207-213

47. Meczekalski, B., Katulski, K., Czyzyk, A., Podfigurna-Stopa, A., and

Maciejewska-Jeske, M. (2014) Functional hypothalamic amenorrhea and its influence on women's health. J. Endocrinol. Invest. 37, 1049-1056 
Table 1. Demographic and anthropometric measures of the study groups.

\begin{tabular}{lcccc}
\hline & $\begin{array}{c}\text { SedOv } \\
\mathbf{n = 1 7}\end{array}$ & $\begin{array}{c}\text { ExOv } \\
\mathbf{n = 1 7}\end{array}$ & $\begin{array}{c}\text { ExFHA } \\
\mathbf{n = 1 1}\end{array}$ & $\begin{array}{c}\text { P value } \\
\text { (main effect) }\end{array}$ \\
\hline Age (years) & $23.5 \pm 0.6$ & $23.5 \pm 1.2$ & $25.4 \pm 1.1$ & 0.312 \\
Height (m) & $1.65 \pm 1.5$ & $1.66 \pm 1.5$ & $1.67 \pm 1.5$ & 0.400 \\
Weight (kg) & $58.6 \pm 1.5$ & $58.0 \pm 1.9$ & $58.7 \pm 2.3$ & 0.953 \\
BMl (kg/m ${ }^{2}$ ) & $21.7 \pm 0.5$ & $20.9 \pm 0.5$ & $20.8 \pm 0.8$ & 0.438 \\
Body fat (\%) & $28.7 \pm 2.3$ & $24.7 \pm 2.0$ & $21.8 \pm 2.2$ & 0.118 \\
Fat free mass (kg) & $38.8 \pm 1.4$ & $42.0 \pm 1.6$ & $44.1 \pm 1.3$ & 0.092 \\
Estradiol (pmol/L) & $130.9 \pm 10.9$ & $148.7 \pm 19.2$ & $87.8 \pm 14.7^{*}$ & 0.007 \\
Progesterone (nmol/L) & $1.7 \pm 0.2$ & $1.8 \pm 0.2$ & $1.7 \pm 0.2$ & 0.895 \\
T3 (pmol/L) & $4.2 \pm 0.2$ & $4.3 \pm 0.1$ & $3.6 \pm 0.6^{\dagger}$ & $<0.001$ \\
Testosterone (nmol/L) & $2.4 \pm 0.2$ & $2.1 \pm 0.2$ & $2.4 \pm 0.2$ & 0.371 \\
SHBG (nmol/L) & $40.5 \pm 2.7^{\ddagger}$ & $52.9 \pm 1.9$ & $51.6 \pm 4.1$ & 0.020 \\
FAl (au) & $0.06 \pm 0.02$ & $0.05 \pm 0.01$ & $0.05 \pm 0.01$ & 0.137 \\
VO ${ }_{2}$ peak (mL/kg $\left.{ }^{-1} / \mathrm{min}^{-1}\right)$ & $38.9 \pm 0.8^{\ddagger}$ & $46.6 \pm 1.3$ & $47.8 \pm 2.0$ & $<0.001$ \\
\hline
\end{tabular}

Values are mean \pm SEM.

BMI, body mass index; FAI, free androgen index; $\mathrm{T} 3$, triiodothyronine; $\mathrm{VO}_{2}$ peak, peak oxygen consumption.

\footnotetext{
*ExFHA vs ExOv

${ }^{\dagger}$ ExFHA vs ExOv and SedOv

‡ SedOr vs ExOv and ExFHA
} 
Table 2. Blood pressure and heart rate responses to graded LBNP.

\begin{tabular}{|c|c|c|c|c|}
\hline & HR & SBP & DBP & MAP \\
\hline \multicolumn{5}{|l|}{ SedOr $^{\dagger}$} \\
\hline $\mathrm{Ba}$ & $61 \pm 2$ & $102 \pm 2$ & $59 \pm 1$ & $74 \pm 1$ \\
\hline$-10 \mathrm{mmHg}$ & $61 \pm 2$ & $98 \pm 2^{\S}$ & $55 \pm 1^{\S}$ & $69 \pm 1^{\S}$ \\
\hline$-20 \mathrm{mmHg}$ & $65 \pm 3$ & $96 \pm 1^{\S}$ & $53 \pm 1^{\S}$ & $67 \pm 2^{\S}$ \\
\hline$-40 \mathrm{mmHg}$ & $80 \pm 3^{\S}$ & $94 \pm 2^{\S}$ & $51 \pm 2^{\S}$ & $66 \pm 2^{\S}$ \\
\hline \multicolumn{5}{|l|}{$\operatorname{ExOV}^{\dagger}$} \\
\hline $\mathrm{Ba}$ & $57 \pm 2$ & $105 \pm 2$ & $61 \pm 1$ & $76 \pm 1$ \\
\hline$-10 \mathrm{mmHg}$ & $58 \pm 2$ & $102 \pm 2^{\S}$ & $57 \pm 2^{\S}$ & $72 \pm 2^{\S}$ \\
\hline$-20 \mathrm{mmHg}$ & $62 \pm 3^{\S}$ & $100 \pm 2^{\S}$ & $54 \pm 2^{\S}$ & $70 \pm 2^{\S}$ \\
\hline$-40 \mathrm{mmHg}$ & $72 \pm 3^{\S}$ & $98 \pm 1^{\S}$ & $53 \pm 2^{\S}$ & $68 \pm 2^{\S}$ \\
\hline \multicolumn{5}{|l|}{$E x F H A^{\dagger}$} \\
\hline $\mathrm{Ba}$ & $47 \pm 2^{*}$ & $94 \pm 2^{*}$ & $58 \pm 2$ & $70 \pm 2$ \\
\hline$-10 \mathrm{mmHg}$ & $48 \pm 2^{*}$ & $93 \pm 2^{*}$ & $56 \pm 2$ & $68 \pm 2$ \\
\hline$-20 \mathrm{mmHg}$ & $49 \pm 2^{*}$ & $92 \pm 2^{*}$ & $53 \pm 2^{\S}$ & $66 \pm 2^{\S}$ \\
\hline$-40 \mathrm{mmHg}$ & $60 \pm 2^{\star \S}$ & $89 \pm 2^{\star \S}$ & $51 \pm 2^{\S}$ & $64 \pm 2^{\S}$ \\
\hline
\end{tabular}

Values are mean \pm SEM.

$\mathrm{Ba}$, baseline; DBP, diastolic BP; HR, heart rate; MAP, mean arterial BP; SBP, systolic BP.

${ }^{\dagger}$ LBNP main effect for HR, SBP, DBP and MAP within each group, $p<0.001$.

* ExFHA versus SedOv and ExOv within condition, $\mathrm{p}<0.05$.

$\S$ Significantly different from baseline within groups, $p<0.05$. 
Table 3. Baseline HRV for the study groups.

\begin{tabular}{lcccc}
\hline & SedOv & ExOv & ExFHA & $\begin{array}{c}\text { P } \\
\text { (main } \\
\text { effect) }\end{array}$ \\
\hline Absolute $\left(\mathrm{ms}^{2}\right)$ & $\mathbf{n = 1 7}$ & $\mathbf{n = 1 7}$ & $\mathbf{n = 1 2}$ & \\
LF & $686 \pm 135$ & $1024 \pm 265$ & $1023 \pm 165$ & 0.398 \\
HF & $837 \pm 122$ & $948 \pm 130$ & $2364 \pm 757^{*}$ & 0.012 \\
VLF & $818 \pm 115$ & $1160 \pm 179$ & $1705 \pm 549$ & 0.114 \\
LF+HF & $1524 \pm 220$ & $1971 \pm 341$ & $3386 \pm 894^{\star *}$ & 0.034 \\
Total (LF+HF+VLF) & $2347 \pm 253$ & $3137 \pm 349$ & $5092 \pm 1310^{*}$ & 0.021 \\
LF/HF & $0.9 \pm 0.1$ & $1.1 \pm 0.2$ & $0.6 \pm .01$ & 0.095 \\
& & & & \\
Normalized (\%) & & & & 0.129 \\
LF (LF/[LF+HF]*100) & $44 \pm 3$ & $46 \pm 5$ & $34 \pm 4$ & 0.129 \\
HF (HF/[LF+HF]*100) & $56 \pm 3$ & $54 \pm 5$ & $66 \pm 4$ & \\
& & & & 0.516 \\
Transformed (log10) & & & & \\
LF & $2.7 \pm 0.1$ & $2.8 \pm .01$ & $3.2 \pm 0.1^{*}$ & 0.022 \\
HF & $2.8 \pm 0.1$ & $2.9 \pm 0.1$ & & \\
\hline
\end{tabular}

Values are mean \pm SEM.

* ExFHA vs SedOv and ExOv, $p<0.05$

** ExFHA vs SedOv, $p<0.05$ 
Figure 1. Chart showing frequency domain indices of heart rate variability (HRV) at baseline and during graded LBNP in SedOv (closed circles), ExOv (closed squares) and ExFHA (open triangles) women. HRV indices include : HF log10 (A), LFlog10 (B), Total HRV (C), and LF/HF (D).

* ExFHA versus SedOv and ExOv, $p<0.05$; ExFHA versus ExOv, p<0.05. L x G, LBNP $x$ Group interaction. 
Figure 1.

A

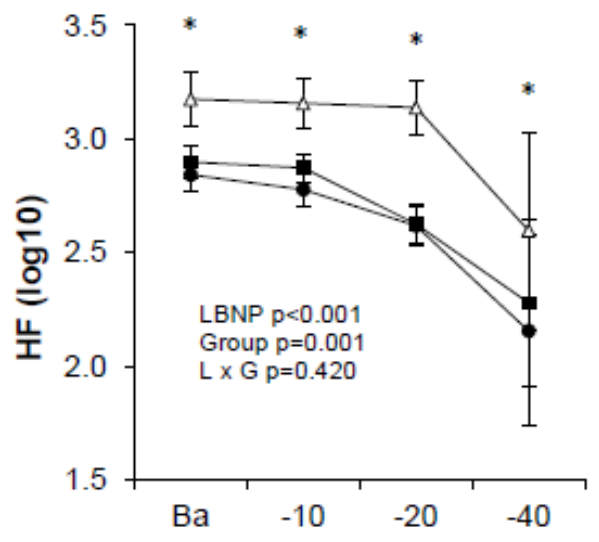

C

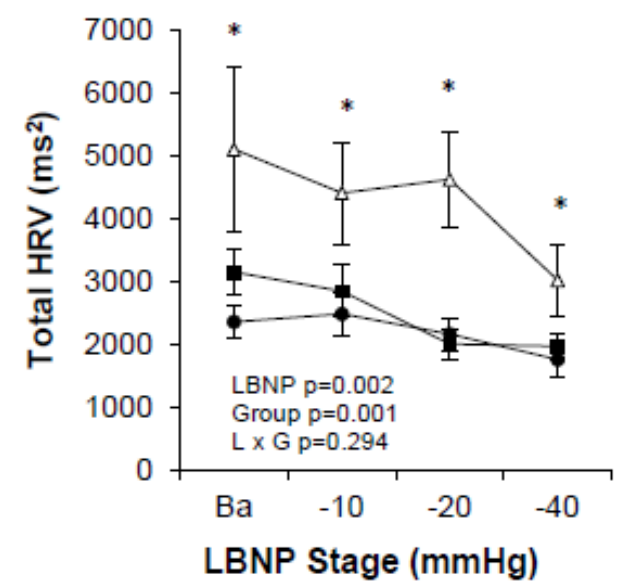

B

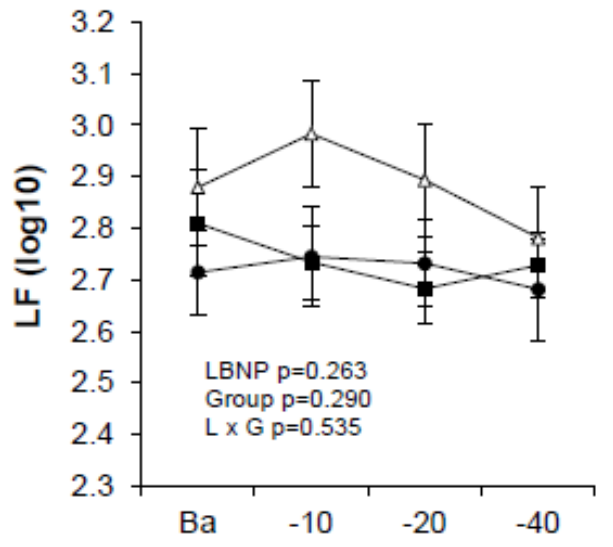

D

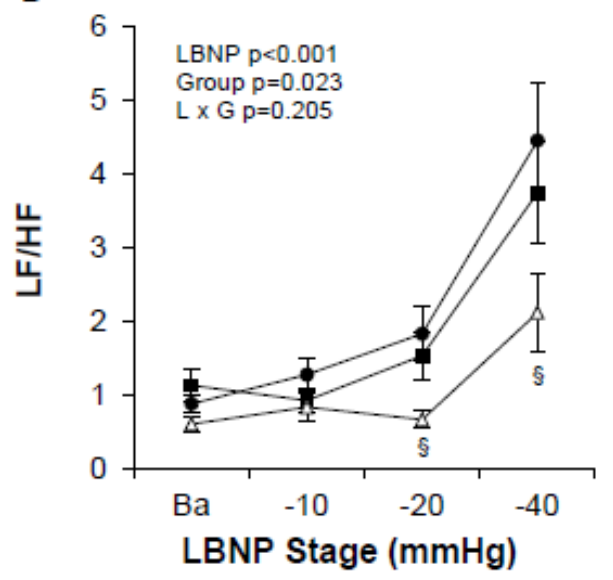

